Annals of Pure and Applied Mathematics

Vol. 16, No. 2, 2018, 365-372

ISSN: 2279-087X (P), 2279-0888(online)

Published on 24 February 2018

Annals of

www.researchmathsci.org

DOI: http://dx.doi.org/10.22457/apam.v16n2a13

\title{
Further Results on Vertex Odd Divisor Cordial Labeling of Some Graphs
}

\author{
A. Sugumaran ${ }^{1}$ and K. Suresh ${ }^{2}$ \\ Department of Mathematics, Government Arts College \\ Tiruvannamalai - 606 603, Tamil Nadu, India. \\ Email: ${ }^{1}$ sugumaranaruna@gmail.com, 2 dhivasuresh@gmail.com \\ ${ }^{2}$ Corresponding author \\ Received 15 January 2018; accepted 13 February 2018
}

Abstract. In this paper we prove that the graphs $\prec F_{n} \boldsymbol{\Lambda} F_{n} \succ$, Theta graph, Switching of a vertex in a Petersen graph, $\prec K_{1, n} \Delta K_{1, n} \Delta K_{1, n} \succ$ and $\prec K_{1, n} \Delta K_{1, n} \mathbf{\Delta} K_{1, n} \mathbf{\Delta} K_{1, n} \succ$ are vertex odd divisor cordial graphs.

Keywords: Graph labeling, cordial labeling, divisor cordial labeling, vertex odd divisor cordial labeling.

\section{AMS Mathematics Subject Classification (2010): 05C78}

\section{Introduction}

Graph theory has several interesting applications in system analysis, operations research and economics. Since most of the time the aspects of graph problems are uncertain, it is nice to deal with these aspects via the methods of labeling. The concept of labeling of graphs is an active research area and it has been widely studied by several researchers. In a wide area network (WAN), several systems are connected to the main server, the labeling technique plays a vital role to label the cables. The labeling of graphs have been applied in the fields such as circuit design, communication network, coding theory, and crystallography.

A graph labeling, is a process in which each vertex is assigned a value from the given set of numbers, the labeling of edges depends on the labels of its end vertices. An excellent survey of various graph labeling problems, we refer to Gallian [2]. Two well known graph labeling methods are graceful labeling and harmonious labeling. These labelings are studied by Cahit [1].

Cordial labeling was introduced by Cahit [1]. Many labeling schemes were introduced with slight variations in cordial such as prime cordial labeling, divisor cordial labeling. Varatharajan et al. [10] have analyzed the divisor cordial labeling.The divisor cordial labeling of various types of graph is presented in [5,6,7,8,11] Muthaiyan et al. [4] introduce the concept of vertex odd divisor cordial graph. In section 2, we summarize the necessary definitions and basic results. In section 3 , we proved that some standard graphs are vertex odd divisor cordial graph. We conclude in section 4 . 


\section{A. Sugumaran and K. Suresh}

\section{Basic definitions}

In this section, we provide a brief summary of the definitions and other results which are prerequisites for the present work.

All the graphs considered here are simple finite, undirected without loops and multiple edges. Let $G=(V, E)$ be a graph and as usual we denote $p=|V|$ and $q=|E|$. For terminology and notations not specifically defined here, we refer to Harary [3].

We recall the following definition from Harary [3].

Definition 2.1. Let $G=(V, E)$ be a graph. A mapping $f: V \rightarrow\{0,1\}$ is called the binary vertex labeling of $G$ and $f(v)$ is called the label of the vertex $v \in V$ of $G$ under $\mathrm{f}$. The induced edge labeling $f^{*}: E \rightarrow\{0,1\}$ is given by $f^{*}(e)=|f(u)-f(v)|$, for all $e=u v \in E$.

We denote $v_{f}(i)$ is the number of vertices of $G$ having label $i$ under $f$ and $e_{f}(i)$ is the number of edges of $G$ having label $i$ under $f$, where $i=0,1$. Now we define cordial labeling of a graph.

Definition 2.2. [1] Let $G=(V, E)$ be a graph and $f: V \rightarrow\{0,1\}$ be a binary vertex labeling of $\mathrm{G}$. The map $f$ is called a cordial labeling if $\left|v_{f}(0)-v_{f}(1)\right| \leq 1$ and $\left|e_{f}(0)-e_{f}(1)\right| \leq 1$.

A graph $G$ is called cordial graph if it admits cordial labeling.

Definition 2.3. [10] A divisor cordial labeling of a graph $G=(V, E)$ is a bijection $f: V \rightarrow\{1,2,3, \ldots,|V|\}$ such that if each edge uv is assigned the label 1 if $f(u) / f(v)$ or $f(v) / f(u)$ and the label 0 if $f(u) f(v)$, then $\left|e_{f}(0)-e_{f}(1)\right| \leq 1$.

Definition 2.4. [4] A vertex odd divisor cordial labeling of a graph $G=(V, E)$ is a bijection $f: V \rightarrow\{1,2,3, \ldots, 2 n-1\}$ such that if each edge uv is assigned the label 1 if $f(u) / f(v)$ or $f(v) / f(u)$ and the label 0 if $f(u) f(v)$, then $\left|e_{f}(0)-e_{f}(1)\right| \leq 1$.

A graph which admits odd divisor cordial labeling is called a vertex odd divisor cordial graph.

Definition 2.5. [9] Consider two copies of graph $G$ namely $G_{1}$ and $G_{2}$. Then the graph $G^{\prime}=\prec G_{1} \Delta G_{2} \succ$ is the graph obtained by joining the apex vertices of $G_{1}$ and $G_{2}$ by an edge as well as to a new vertex $v^{\prime}$.

Note that $\prec G_{1} \Delta G_{2} \Delta G_{3} \succ=\prec\left(G_{1} \Delta G_{2}\right) \Delta G_{3} \succ$ and

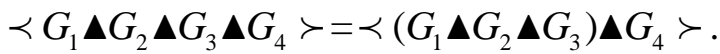

Definition 2.6. The friendship graph $F n$ is a planar undirected graph with $2 n+1$ vertices and $3 \mathrm{n}$ edges. The friendship graph Fn can be constructed by joining $\mathrm{n}$ copies of the cycle 
Further Results On Vertex Odd Divisor Cordial Labeling of Some Graphs graph $C_{3}$ with a common vertex.

Definition 2.7. A vertex switching $G_{v}$ of a graph $G$ is the graph obtained by taking a vertex $v$ of $G$, removing all the edges incident to $v$ and adding edges joining $v$ to every other vertex which are not adjacent to $v$ in $G$.

\section{Main results}

Theorem 3.1. The graph $G=\prec F_{n} \Delta F_{n} \succ$ is a vertex odd divisor cordial graph.

Proof: Let $G_{1}=G_{2}=F_{n}$. Let $G$ be the graph $\prec G_{1} \mathbf{\Delta} G_{2} \succ$. Let $u$ and $v$ be the apex vertices of $G_{1}$ and $G_{2}$. Let Let $u_{1}, u_{2}, u_{3}, \ldots, u_{2 n}$ be the vertices of $G_{1}$ and $v_{1}, v_{2}, v_{3}, \ldots, v_{2 n}$ be the vertices of $G_{2}$ respectively. Let $v^{\prime}$ be the new vertex joining with the apex vertices $u$ and $v$. Then $|V(G)|=4 n+3$ and $|E(G)|=6 n+3$. We define $f: V(G) \rightarrow\{1,3,5, \ldots, 8 n+5\}$, as follows $f(u)=1, f(v)=3 f\left(v^{\prime}\right)=p$ where $p$ is the largest prime number such that $0 \prec p \leq 8 n+5$.

$$
f\left(v_{i}\right)=\left\{\begin{array}{rll}
9+6(i-1) & \text { if } & 1 \leq i \leq n \\
11+6(i-n-1) & \text { if } & n+1 \leq i \leq 2 n
\end{array}\right.
$$

The remaining labels are assigned to the vertices $u_{1}, u_{2}, u_{3}, \ldots, u_{2} n$ in ascending order. From the above labeling pattern, we have $e_{f}(0)=3 n+1$ and $e_{f}(1)=3 n+2$. Therefore $\left|e_{f}(0)-e_{f}(1)\right| \leq 1$.

Hence, $G$ is a vertex odd divisor cordial graph.

Example 3.2. Vertex odd divisor cordial labeling of the graph $\prec F_{7} \Delta F_{7} \succ$ is shown in Figure 1.

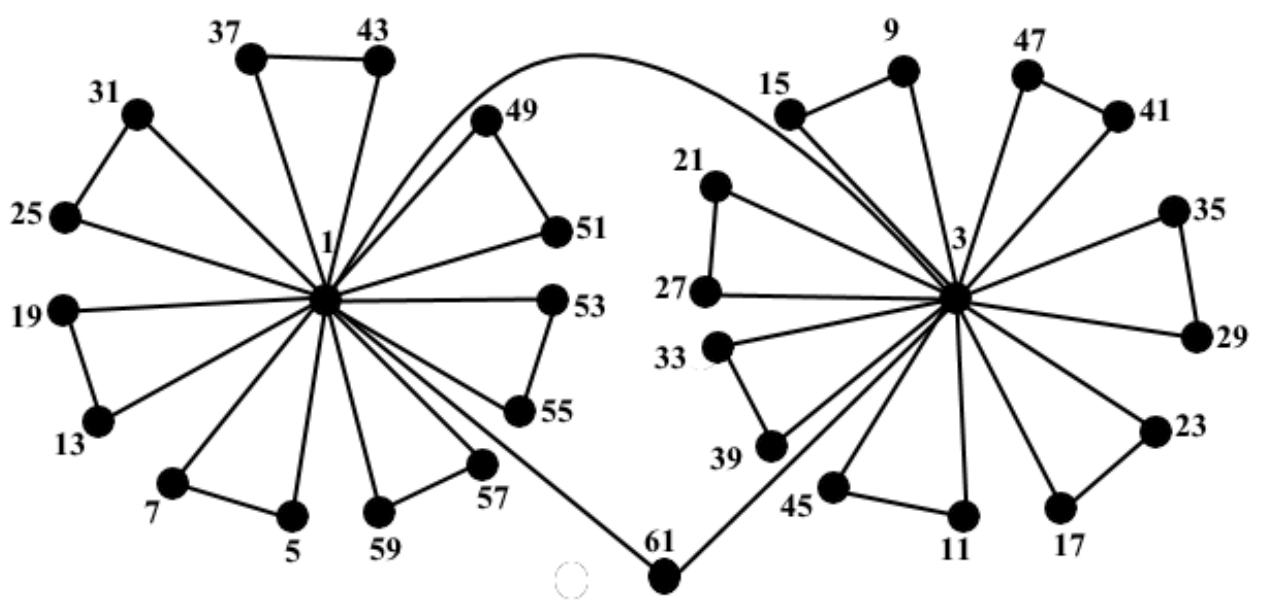

Figure 1: Vertex odd divisor cordial labeling of $\prec F_{7} \Delta F_{7} \succ$ 


\section{A. Sugumaran and K. Suresh}

Theorem 3.3. Theta graph is a vertex odd divisor cordial graph.

Proof: Let $v_{1}, v_{2}, v_{3}, v_{4}, v_{5}, v_{6}$ be the vertices of Theta graph. Let $G$ be the Theta graph. Then $|V(G)|=6$ and $|E(G)|=7$.

We define $f: V(G) \rightarrow\{1,3,5,7,9,11\}$ as follows

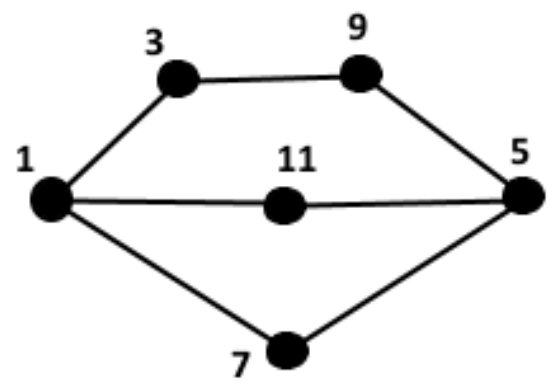

Figure 2: Vertex odd divisor cordial labeling of theta graph $f\left(v_{1}\right)=1, f\left(v_{i+1}\right)=3+6(i-1), 1 \leq i \leq 2$.

Except the labels assigned from the set $\{1,3,5, \ldots, 11\}$ the remaining labels are assigned to $v_{4}, v_{5}, v_{6}$ in any order.We observe that, from the above labeling pattern, we have $e_{f}(1)=4$ and $e_{f}(0)=3$. Therefore $\left|e_{f}(0)-e_{f}(1)\right| \leq 1$.

Hence $\mathrm{G}$ is a vertex odd divisor cordial graph.

Theorem 3.4. Switching of a vertex in a Petersen graph admits vertex odd divisor cordial graph.

Proof: Let $G$ be the Petersen graph and let $V(G)=\left\{v, v_{i}: 1 \leq i \leq 9\right\}$ be the vertex set.

Let $G_{v}$ be the graph obtained from $G$ by switching the vertex $v$.

Then $\left|V\left(G_{v}\right)\right|=10$ and $\left|E\left(G_{v}\right)\right|=18$.

We define $f: V(G) \rightarrow\{1,3,5, \ldots 19\}$, as follows

$f(v)=1, f\left(v_{i}\right)=3+6(i-1), 1 \leq i \leq 2 f\left(v_{3}\right)=7, f\left(v_{2 i+2}\right)=5+10(i-1), 1 \leq i \leq 2$.

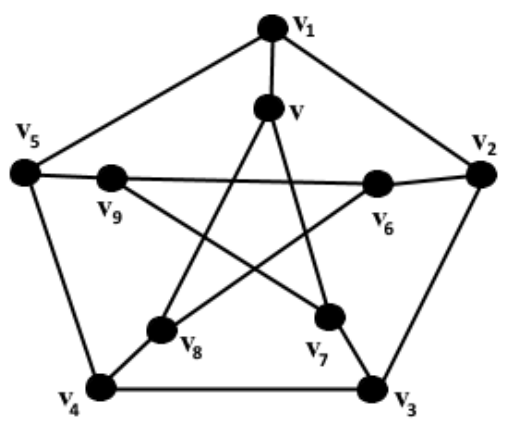

Figure 3: Petersen graph 
Further Results On Vertex Odd Divisor Cordial Labeling of Some Graphs

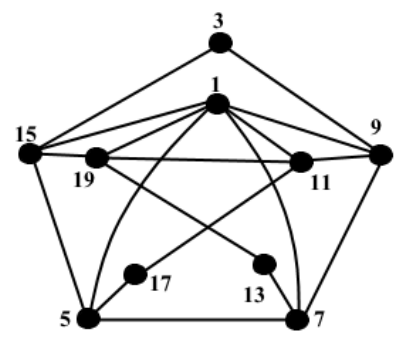

Figure 4: Switching of a vertex $v$ in Petersen graph

Except the labels assigned from the set $\{1,3,5, \ldots, 19\}$ the remaining labels are assigned to $\left\{v_{6}, v_{7}, v_{8}, v_{9}, v_{10}\right\}$ in any order.

In view of above defined labeling pattern, we have $e_{f}(0)=e_{f}(1)=9$.

Therefore $\left|e_{f}(0)-e_{f}(1)\right| \leq 1$.

Hence $G$ is a vertex odd divisor cordial graph.

Theorem 3.5. The graph $G=\prec K_{1, n} \Delta K_{1, n} \Delta K_{1, n} \succ$ is a vertex odd divisor cordial graph. Proof: Let $G_{1}=G_{2}=G_{3}=K_{1, n}$. Let $G$ be the graph $G=\prec K_{1, n} \mathbf{\Delta} K_{1, n} \mathbf{\Delta} K_{1, n} \succ$. Let $u, v$ and $w$ be the apex vertices of $G_{1}, G_{2}$ and $G_{3}$. Let $\left\{u_{i}, v_{i}, w_{i}: 1 \leq i \leq n\right\}$ be the pendant vertices, where $u_{i}, v_{i}, w_{i}(1 \leq i \leq n)$ are attached with $u, v$ and $w$ respectively. Let $v^{\prime}$ and $v^{\prime \prime}$ be the new vertices joining with apex vertices $u$ and $v, v$ and $w$ respectively. Then $|V(G)|=3 n+5$ and $|E(G)|=3 n+6$.

We define $f: V(G) \rightarrow\{1,3,5, \ldots, 6 n+9\}$, as follows $f(u)=p, f(v)=1, f(w)=3, f\left(v^{\prime}\right)=7, f\left(v^{\prime \prime}\right)=5$ where $p$ is the largest prime number such that $0 \prec p \leq 6 n+9$.

case: 1 For $n=2$.

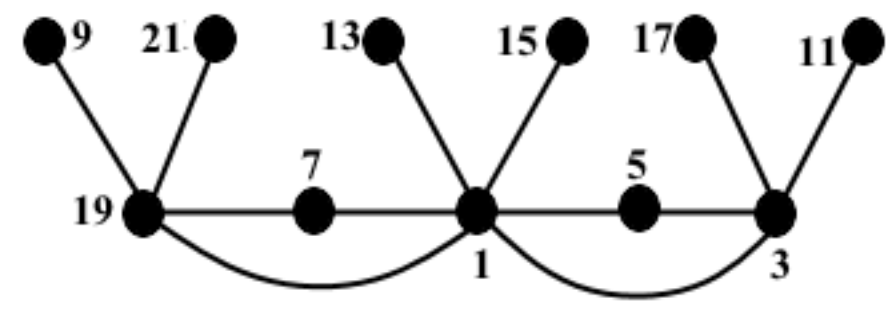

Figure 5: Vertex odd divisor cordial labeling of $\prec K_{1,2} \boldsymbol{\Delta} K_{1,2} \Delta K_{1,2} \succ$

From the above figure we observe that $e_{f}(0)=6=e_{f}(1)$.

Hence $\prec K_{1,2} \boldsymbol{\Delta} K_{1,2} \boldsymbol{\Delta} K_{1,2} \succ$ is a vertex odd divisor cordial graph.

Case 2: when $n$ is even $(n \succ 2)$ 


\section{A. Sugumaran and K. Suresh}

$$
f\left(v_{i}\right)=\left\{\begin{array}{cc}
9+6(i-1) & \text { if } \quad 1 \leq i \leq \frac{n-2}{2} \\
11+6\left(i-\frac{n}{2}\right) & \text { if } \quad \frac{n}{2} \leq i \leq n
\end{array}\right.
$$

Case 3: when $\mathrm{n}$ is odd

$$
f\left(v_{i}\right)=\left\{\begin{array}{c}
9+6(i-1) \quad \text { if } \quad 1 \leq i \leq \frac{n-1}{2} \\
11+6\left(i-\frac{n-1}{2}\right) \text { if } \quad \frac{n+1}{2} \leq i \leq n
\end{array}\right.
$$

Except the labels assigned from the set $\{1,3,5, \ldots, 6 n+9\}$ the remaining labels are assigned to $u_{i}, v_{i}(1 \leq i \leq n)$ in any order.

In view of above defined labeling pattern, we have

$$
\begin{aligned}
& e_{f}(1)=\left\{\begin{array}{c}
3 n \text { if } \mathrm{n} \text { is even } \\
3 n-1 \text { if } \mathrm{n} \text { is odd and }
\end{array}\right. \\
& e_{f}(0)=\left\{\begin{array}{c}
3 n \text { if } \mathrm{n} \text { is even } \\
3 n-2 \text { if } \mathrm{n} \text { is odd }
\end{array}\right.
\end{aligned}
$$

Therefore $\left|e_{f}(0)-e_{f}(1)\right| \leq 1$.

Hence $G$ is vertex odd divisor cordial labeling graph.

Example 3.6. Vertex odd divisor cordial labeling for $\prec K_{1,7} \boldsymbol{\Delta} K_{1,7} \boldsymbol{\Delta} K_{1,7} \succ$ and $\prec K_{1,8} \boldsymbol{\Delta} K_{1,8} \boldsymbol{\Delta} K_{1,8} \succ$ are shown in Figures 6 and 7 .

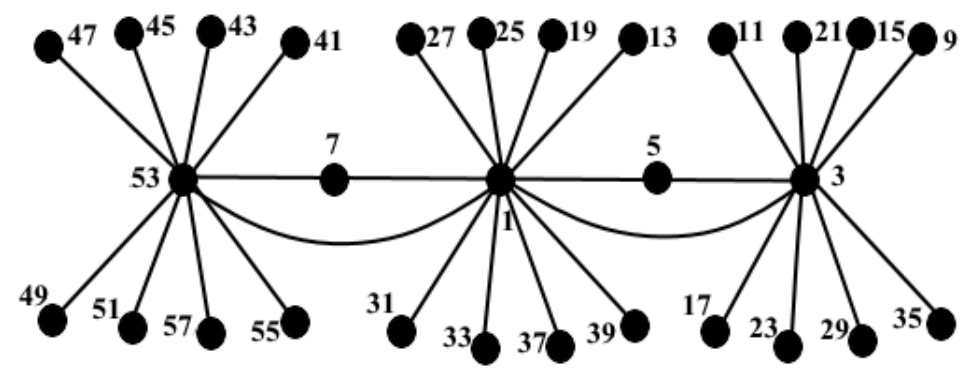

Figure 6: Vertex odd divisor cordial labeling of $\prec K_{1,7} \boldsymbol{\Delta} K_{1,7} \boldsymbol{\Delta} K_{1,7} \succ$ 
Further Results On Vertex Odd Divisor Cordial Labeling of Some Graphs

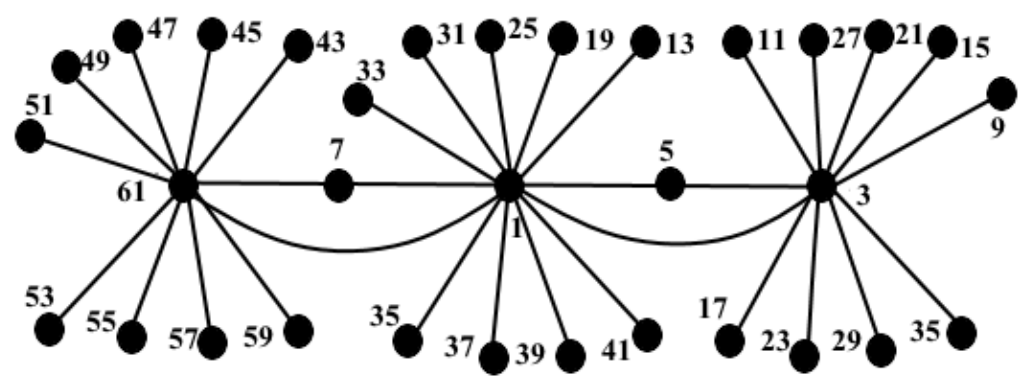

Figure 7: Vertex odd divisor cordial labeling of $\prec K_{1,8} \Delta K_{1,8} \mathbf{\Delta} K_{1,8} \succ$

Theorem 3.7. The graph $G=\prec K_{1, n} \Delta K_{1, n} \Delta K_{1, n} \Delta K_{1, n} \succ$ is vertex odd divisor cordial graph.

Proof: Let $G_{1}=G_{2}=G_{3}=G_{4}=K_{1, n}$. Let $G$ be the graph $G=\prec K_{1, n} \boldsymbol{\Delta} K_{1, n} \mathbf{\Delta} K_{1, n} \Delta K_{1, n} \succ$. Let $u_{1}, u_{2}, u_{3}$ and $u_{4}$ be the apex vertices of $G_{1}$, $G_{2}, G_{3}$ and $G_{4}$. Let $\left\{u_{1}^{i}, u_{2}^{i}, u_{3}^{i}, u_{4}^{i}: 1 \leq i \leq n\right\}$ be the pendant vertices are attached with $u_{1}, u_{2}, u_{3}$ and $u_{4}$ respectively. Let $v^{\prime}, v^{\prime \prime}$ and $v^{\prime \prime \prime}$ be the new vertices joining with apex vertices $u_{1}$ and $u_{2}, u_{2}$ and $u_{3}, u_{3}$ and $u_{4}$ respectively.

Then $|V(G)|=4 n+7$ and $|E(G)|=4 n+9$.

We define $f: V(G) \rightarrow\{1,3,5, \ldots, 8 n+13\}$ as follows

$$
\begin{aligned}
& f\left(u_{1}\right)=p_{1}, \\
& f\left(u_{2}\right)=1, \\
& f\left(u_{3}\right)=3, \\
& f\left(u_{4}\right)=p_{2}, \\
& f\left(v^{\prime}\right)=11, \\
& f\left(v^{\prime \prime}\right)=7, f\left(v^{\prime \prime \prime}\right)=5
\end{aligned}
$$

where $p_{1}$ and $p_{2} \quad\left(p_{1} \neq p_{2}\right)$ are the largest prime number such that $0 \prec p_{2} \prec p_{1} \leq 8 n+13$. $f\left(u_{3}^{i}\right)=9+6(i-1) ; 1 \leq i \leq n$ Except the labels assigned from the set $\{1,3,5, \ldots, 8 n+13\}$ the remaining labels are assigned to $u_{1}^{i}, u_{2}^{i}, u_{4}^{i}(1 \leq i \leq n)$ in any order.

In view of above defined labeling pattern, we have $e_{f}(0)=2 n+5$ and $e_{f}(1)=2 n+4$.

Therefore $\left|e_{f}(0)-e_{f}(1)\right| \leq 1$. Hence $G$ is vertex odd divisor cordial labeling graph.

Example 3.8. Vertex odd divisor cordial labeling for $\prec K_{1,8} \Delta K_{1,8} \Delta K_{1,8} \Delta K_{1,8} \succ$ is shown in Figure 8. 


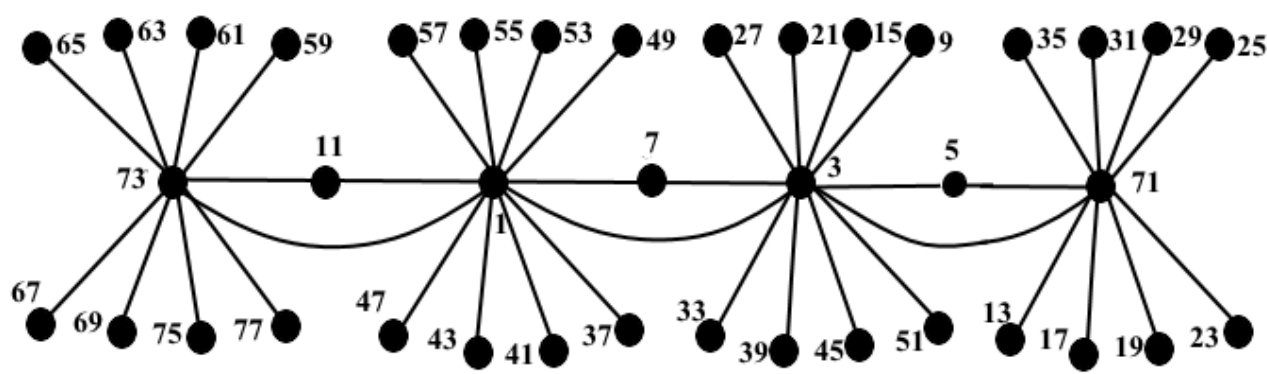

Figure 8: Vertex odd divisor cordial labeling of $\prec K_{1,8} \Delta K_{1,8} \Delta K_{1,8} \Delta K_{1,8} \succ$

\section{Conclusion}

The vertex odd divisor cordial labeling is a variation of cordial labeling. It is very interesting to study graph or families of graph which are vertex odd divisor cordial as all the graphs do not admit vertex odd divisor cordial labeling. In this paper we proved that the graphs $\prec F_{n} \boldsymbol{\Delta} F_{n} \succ$, Theta graph, Switching of a vertex in a Petersen graph, $\prec K_{1, n} \Delta K_{1, n} \mathbf{\Delta} K_{1, n} \succ$ and $\prec K_{1, n} \mathbf{\Delta} K_{1, n} \Delta K_{1, n} \Delta K_{1, n} \succ$ are vertex odd divisor cordial graphs.

\section{REFERENCES}

1. I.Cahit, Cordial graphs: a weaker version of graceful and harmonious graphs, Ars Combinatoria, 23 (1987) 201-207.

2. J.A.Gallian, A dynamic survey of graph labeling, The Electronic Journal of Combinatorics, 16 (2015) \# DS6.

3. F.Harary, Graph Theory, Addison-Wesley, Massachusetts, 1972.

4. A.Muthaiyan and P.Pugalenthi, Vertex odd divisor cordial graphs, International Journal of Innovative Science, Engineering and Technology, 2(10) (2015) 395-400.

5. A.Sugumaran and K.Rajesh, Some new results on sum divisor cordial graphs, Annals of Pure and Applied Mathematics, 14(1) (2017) 45-52.

6. A.Sugumaran and K. Rajesh, Sum divisor cordial labeling of theta graph, Annals of Pure and Applied Mathematics, 14(2) (2017) 313-320.

7. A.Sugumaran and K.Suresh, Star related vertex odd divisor cordial labeling of graphs, Annals of Pure and Applied Mathematics, Communicated.

8. S.K.Vaidya and N.H.Shah, Further results on divisor cordial labeling, Annals of Pure and Applied Mathematics, 4(2) (2013) 150-159.

9. S.K.Vaidya and C.M.Barasara, Product cordial graphs in the context of some graph operations, International Journal of Mathematics and Scientific Computing, 1(2) (2011) 1-6.

10. R.Varatharajan, S.Navaneethakrishnan and K.Nagarajan, Divisor cordial graphs, International Journal of Mathematical Combinatorics, 4 (2011) 15-25.

11. R.Varatharajan, S.Navaneethakrishnan and K.Nagarajan, Special classes of divisor cordial graphs, International Mathematical Forum, 7(35) (2012) 1737-1749. 